\title{
Normal Mode Determination of Perovskite Crystal Structures with Octahedral Rotations: Theory and Applications
}

\author{
Mohammad A. Islam, James M. Rondinelli, and Jonathan E. Spanier* \\ Department of Materials Science \& Engineering, Drexel University, Philadelphia, PA 19104, USA
}

(Dated: March 5, 2013)

\begin{abstract}
Nuclear site analysis methods are used to enumerate the normal modes of $A B X_{3}$ perovskite polymorphs with octahedral rotations. We provide the modes of the fourteen subgroups of the cubic aristotype describing the Glazer octahedral tilt patterns, which are obtained from rotations of the $B X_{6}$ octahedra with different sense and amplitude about high symmetry axes. We tabulate all normal modes of each tilt system and specify the contribution of each atomic species to the mode displacement pattern, elucidating the physical meaning of the symmetry unique modes. We have systematically generated 705 schematic atomic displacement patterns for the normal modes of all 15 (14 rotated +1 unrotated) Glazer tilt systems. We show through some illustrative examples how to use these tables to identify the octahedral rotations, symmetric breathing, and first-order Jahn-Teller anti-symmetric breathing distortions of the $B X_{6}$ octahedra, and the associated Raman selection rules. We anticipate that these tables and schematics will be useful in understanding the lattice dynamics of bulk perovskites and would serve as reference point in elucidating the atomic origin of a wide range of physical properties in synthetic perovskite thin films and superlattices.
\end{abstract}

PACS numbers: 61.50.Ah, 61.68.+n, 63.20.-e, 63.20.D-

\section{INTRODUCTION}

The compounds with the general formula $A B X_{3}(X=\mathrm{O}$ or a halogen) where $A$ is either an (alkaline) earth or a rare earth metal and $B$ is a transition metal with partly filled $d$-orbitals form the perovskite crystal family. Perovskites are a truly versatile material class that is malleable both chemically and structurally. At least $30 \%$ of the elements from the periodic table can occupy the $A$ cation position and over half of the periodic table fills the $B$ cation position, with almost $100 \%$ substitution. ${ }^{1}$ The structural adaptability of the $A B X_{3}$ perovskites, enabled by the flexible and corner-connected $B \mathrm{X}_{6}$ octahedral network, explains why such diverse chemistries are compatible in the perovskite crystal structure (Fig. 1).

The chemical and structural compatibility of oxidebased perovskites at the atomic-scale makes it possible to tune their macroscopic properties in a variety of ways, e.g., by judicious choice of the cations (chemical pressure) ${ }^{2,3}$ and hydrostatic pressure. ${ }^{4}$ Consider the perovskite oxide series $A \mathrm{NiO}_{3}$, where $A$ is a rare-earth element: The Ni-O-Ni bond angles between neighboring octahedra can be made to approach $180^{\circ}$ (cubic symmetry), driving an insulator-metal transition as the $A$-site ionic radius is increased systematically by substitution of $\mathrm{Eu}, \mathrm{Nd}, \mathrm{Pr}$ and La. ${ }^{5}$ A modern technique to tune structure-derived electronic properties in perovskites includes octahedral engineering, where substrate-imposed strain and superlattice formation are used to alter the magnitude and flavor of the $\mathrm{BO}_{6}$ octahedral rotation patterns by exploiting both misfit strain-octahedral rotation and heterointerfacial coupling between two similar or dissimilar tilt patterns possessed by the substrate and film. ${ }^{6,7}$ The directed control of the crystal structure in epitaxial films often stabilizes states which are not found in bulk equilibrium phases, producing dramatic changes in their macroscopic properties.

Control over macroscopic properties requires an understanding of the atomic structure and an appropriately facile technique to measure both the property-dictating structural units - in this case the $\mathrm{BO}_{6}$ octahedra - and their response to external stimuli. In other words, appropriate experimental methods are required to determine the structural transitions in functional perovskite oxides induced by hydrostatic or chemical pressures, strain and superlattice formation. However, the measurement of oxygen positions in ultrathin $A B \mathrm{O}_{3}$ films, required to identify the octahedral tilt patterns, is non-trivial for a variety of experimental reasons: ${ }^{8}$ Thin films present limited sample volume and often are grown on relatively thick substrates, which in addition to weak scattering from the oxygen atoms, makes structure determination of the film challenging. Raman spectroscopies, along with real and reciprocal space imaging, ${ }^{9,10}$ however, are experimental techniques that can reliably determine octahedral tilt patterns.

Raman scattering by zone-center, $k=(0,0,0)$, phonons is an established method for identifying structural phases in solid, thin-film, and nanostructured semiconductor and oxide materials, since vibrational modes provide a unique signature of the crystal structure. Analyses of the appearance, energies, relative intensities, linewidths and lineshapes, and symmetry of Raman peaks collected have shown Raman scattering to be indispensable for investigating important relationships among correlated structural, electronic charge and spin, and dipolar degrees of freedom in complex oxide materials. ${ }^{11}$ Despite the optical diffraction-limited spatial resolution, Raman scattering can probe the onset of a new phase with local correlation lengths of a few unit cells. Furthermore, judicious use of the optical polarization vectors' relationship with respect to the plane of the sample, which is 
obtained through cross sectional sample preparation, can selectively enhance the Raman scattering intensity by selected phonons, particularly in the vicinity of a phase phase transition.

Raman spectroscopy studies on cubic $A B \mathrm{O}_{3}$ type perovskite systems (e.g., $\mathrm{BaTiO}_{3}$ ) have been carried out by DiDomenico et al. and Scott, focusing on the the splitting of the Raman active modes due to the cubicto-tetragonal transformation in these systems. ${ }^{12,13}$ More recently, Raman spectroscopy on $A B \mathrm{O}_{3}$ type perovskite oxides have focused on the orthorhombic and the rhombohedral structures ${ }^{14,15}$ The primary displacement patterns investigated in these studies include $(i)$ the soft modes associated with the rotation of the rigid $\mathrm{BO}_{6}$ octahedra about different crystallographic axes, and (ii) the higher energy symmetric and anti-symmetric octahedral breathing-type modes associated with first-order JahnTeller effects.

Dubroka et al. have shown in $\mathrm{La}_{1-y} \mathrm{Sr}_{y} \mathrm{Mn}_{1-x} M_{x} \mathrm{FeO}_{3}$ $(M=\mathrm{Cr}, \mathrm{Co}, \mathrm{Cu}, \mathrm{Zn}, \mathrm{Sc}$ or $\mathrm{Ga}$ ) that the frequency of the soft mode evolves linearly with the octahedral rotation angle about the [110]-pseudocubic direction with a positive slope of $20 \mathrm{~cm}^{-1}$ per degree-rotation. ${ }^{16}$ Since the oxygen positions dictate the octahedral tilt angle, Raman scattering provides a non-destructive and readily accessible means to determine the oxygen positions in $A B \mathrm{O}_{3}$ perovskites, for which, alternative experimental methods require substantial investments in sample preparation, equipment and/or data processing time.

Higher-frequency modes - the Jahn-Teller activated antisymmetric breathing modes and the symmetric breathing modes - often soften across electronic transitions. For this reason, monitoring the evolution in Raman modes associated with breathing-type octahedral distortions can shed light on the origin and onset of charge ordering metal-insulator transitions in bulk perovskites or superlattices formed by interleaving such materials. ${ }^{17}$ In some orthorhombic structures, the Jahn-Teller activated modes are compatible with the crystal structure, i.e., there is no symmetry change, due to two different types of $B$ $\mathrm{O}$ bond lengths induced by the mode. In contrast, the identical $B-\mathrm{O}$ bonds present in rhombohedral structures would require that the Jahn-Teller activated modes reduce the crystal symmetry - the appearance of these modes in a Raman spectrum would reveal a symmetry-breaking phase transition.

The combined sensitivity of Raman spectroscopy to symmetry, structural lattice dynamics and correlated electronic degrees of freedom, and versatility (e.g., facile application of fields and temperature) makes the technique well-suited for probing the capabilities of newly identified octahedral engineering approaches. ${ }^{6,8}$ Reports on Raman scattering selection rules and experimental work on perovskite structures realized through octahedral engineering, however, are rare. In this work we present a comprehensive list of the normal modes, their vector displacement patterns, and the associated selection rules for the 15 octahedral rotation patterns of the $A B \mathrm{O}_{3}$ per-
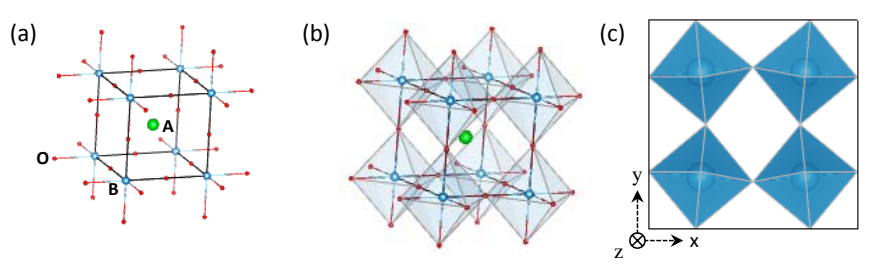

(d)
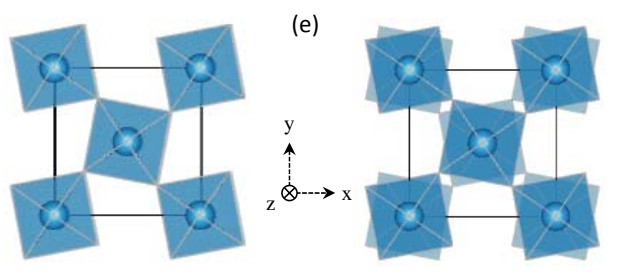

FIG. 1. (Color online) The crystal structure of perovskite oxides with the $A B \mathrm{O}_{3}$ stoichiometry (a) The same structure as in (a) but showing the $\mathrm{BO}_{6}$ octahedral network (b). Schematic illustration of the the $a^{0} b^{+} b^{+}$structure (c), indicating that adjacent octahedra within the same plane rotate in opposite sense to maintain corner connectivity. Schematic illustration of the (d) $a^{0} a^{0} c^{+}$and (e) $a^{0} a^{0} c^{-}$rotation patterns. The inphase rotations and out-of-phase rotations of the octahedra about the $z$-axis are discernible in (d) and (e), respectively. [Note that for clarity the A-site cations and the oxygen atoms are omitted from panels (c), (d) and (e)].

ovskite system (Glazer systems). We have systematically generated 705 schematic atomic displacement patterns for the normal modes of all 15 (14 rotated +1 unrotated) Glazer tilt systems. Furthermore, we have shown how some recent experimental findings can be analyzed using our results and the schematic diagrams to draw decisive conclusions. Our results should be applicable immediately to the complete range of bulk $A B \mathrm{O}_{3}$ perovskites with octahedral tilting. We further anticipate that equipped with comprehensive knowledge of the parent materials one should be able to analyze and rationally design more complex structures (e.g., epitaxial films and superlattices) composed of these $A B \mathrm{O}_{3}$ perovskites.

\section{STRUCTURAL DISTORTIONS IN PEROVSKITES}

The $A B \mathrm{O}_{3}$ type perovskite is built from basic building units of $B_{0}$ octahedra that are corner-connected with additional charge balancing $A$-site cations occupying interstitial positions (Fig. 1). The ideal, aristotype structure, is simple cubic with space group $O_{h}^{1}$ or equivalently $\operatorname{Pm} \overline{3} m$ in the Hermann-Mauguin notation. The $A$ and $B$ cations occupy sites with full cubic symmetry (point group $O_{h}$ ), whereas the $\mathrm{O}$ anions occupy sites with $D_{4 h}$ symmetry. In the ideal structure the ionic radii $r_{A}$, $r_{B}$ and $r_{\mathrm{O}}$ are geometrically related by a characteristic tolerance factor $t=\left(r_{A}+r_{O}\right) / \sqrt{2}\left(r_{B}+r_{O}\right)=1 .{ }^{18}$ The majority of the perovskites, however, are not cubic. The ionic radii of $A, B$ and $\mathrm{O}$ rarely satisfy the condition that 
$t=1$. As a result, the ideal cubic structure undergoes distortions: Typical distortions are rotations of the $\mathrm{BO}_{6}$ octahedra about one or more high-symmetry axes, (anti)parallel displacement of the $A$ or $B$ cations away from their cubic site symmetry, or some combination thereof.

The $A B \mathrm{O}_{3}$ type perovskites can also deviate from the ideal cubic structure by distortions to the rigid $\mathrm{BO}_{6}$ octahedra, i.e., by changes in $B-\mathrm{O}$ bonds lengths. These types of distortions are induced by bond valence requirements, orbital degeneracies, polar distortions and/or valence fluctuations. Changes in $B-\mathrm{O}$ bond lengths, for example, are possible and expected in systems with $d^{4}$ or $d^{7}$ electronic configurations on the $\mathrm{B}$ atoms, leading to Jahn-Teller type antisymmetric breathing of the $B \mathrm{O}_{6}$ octahedra.

The issue of the distortion of the octahedra driven by steric constraints requires special discussion. In the aristotype $P m \overline{3} m$, the octahedra are necessarily regular. The other polymorphs allow distortions and, in general, occur in real systems. Stokes et al. showed that under certain conditions, however, the geometry may not require them. ${ }^{19}$ Consider the simple tilt system $a^{0} a^{0} c^{+}$(No. 127, $\mathrm{P} 4 / \mathrm{mbm}$ ); this system permits octahedral distortions, but does not necessitate them. If the octahedra are rotated by angle $\phi$ around the $z$ axis, then it can be shown that the octahedra will be regular provided $a / c=\sqrt{2} \cos \phi$.

If the ratio of a/c differs from this (and in general it will), the octahedra will be either axially elongated or compressed. Nonetheless, the bond length distortions are almost always compatible with the symmetry reduction induced by the antiferrodistortive rotations. Indeed, Stokes et al. examined the geometrical constrains for the octahedral distortions and found that among the 15 perovskite systems with simple rotation patterns, only one tilt pattern (space group $P 4_{2} / n m c$, no. 137) requires distortions to the octahedra, i.e., it cannot be accommodated without changes in the $B-\mathrm{O}$ bond lengths. ${ }^{19} \mathrm{In}$ general, departure from the cubic symmetry to a structure of lower symmetry will occur with both rigid rotations of octahedra and bond length distortions as the latter, secondary modes, often produce further energy stabilization of the crystal structure.

\section{A. Octahedral Rotation Syntax}

The classification of perovskite crystals according to the rotation patterns of rigid $B \mathrm{O}_{6}$ octahedra is attributed to Glazer. ${ }^{2,3,19-22}$ The corner-connectivity of the $\mathrm{BO}_{6}$ octahedra in the perovskite structure constrains the rotations of the adjacent octahedra. The rotations of an octahedron about a given axis requires that successive octahedra along directions perpendicular to that axis rotate in the opposite sense about that axis. Fig. 1(c) shows that a positive rotation (e.g., counterclockwise) of an octahedron about the $\hat{z}$-axis with magnitude $a$ results in a negative (clockwise) rotation about $\hat{z}$ of equal magnitude of the adjacent octahedra located along the $\hat{x}$ and $\hat{y}$ directions. However, successive octahedra along the rotation axis, $\hat{z}$, can have either the same or opposite rotation sense [Fig. 1(d) and 1(e)].

To simplify and generalize this description, Glazer introduced a notation whereby rotations in perovskites are classified according to how adjacent octahedra rotate along a particular Cartesian axis passing through the $B$ site: $a^{-}\left(a^{+}\right)$indicates out-of-phase (in-phase) rotations while $a^{0}$ denotes no rotations. For example, in a perovskite with the rotation pattern $a^{+} b^{-} b^{-}$the adjacent octahedra along the $x$-axis rotate in phase (e.g., all clockwise) and the adjacent octahedra along the $y$ and $z$ axes rotate out of phase by the same angle $b$. Examples of the two single tilt systems, $a^{0} a^{0} c^{+}$and $a^{0} a^{0} c^{-}$, are shown schematically in Fig. 1(d) and 1(e).

Rotations of the octahedra double the repeat distances perpendicular to the rotation axis and can result in unit cell vectors inclined to each other by angles other than $90^{\circ}$ due to ferroelastic strains. ${ }^{23}$ The transition into the distorted phase changes the number of formula units in the unit cell and the full space group symmetry of the crystal system. By considering all possible rotation senses and amplitudes along various Cartesian directions, Glazer determined that there are a total of 23 different possible rotation patterns. (They are also called tilt systems in the literature.) Some of the tilt systems yield identical space group symmetries: Group-theoretical analysis by Howard and Stokes ${ }^{19}$ shows that of the 23 tilt systems, 15 simple rotation patterns produce 15 centrosymmetric space groups. Table I shows these 15 tilt systems with the appropriate Glazer symbols, the space group notations (and numbers in parentheses), the number of formula units $(Z)$, the point symmetry, and known example compounds. The eight tilt systems that are absent from the group theoretical analysis were found to have higher symmetry than the corresponding space groups. Exhaustive studies by Woodward show that 12 of the 15 tilt systems reported by Howard and Stokes naturally exist(Table I). None of the eight Glazer tilt systems omitted by Howard and Stokes have been experimentally observed. ${ }^{3}$

\section{CRYSTAL SYMMETRY: POINT GROUPS AND SPACE GROUPS}

Before describing the method for normal mode and selection rule determination in the Glazer systems, we provide a brief discussion of point groups, space groups and the relevant symmetry issues pertinent to this analysis.

Crystallographic Point Groups.-The description of a physical crystal requires describing the underlying Bravais lattice - an infinite array of discrete points that appears identical from every point of the array - and the arrangement of atoms or molecular building blocks within a primitive cell. Each crystal system is characterized by a set of rigid operations that takes the system onto itself. This set of operations is known as the symmetry group of the crystal system and includes all translations through 
TABLE I. The Glazer systems found in perovskites. The space group symmetries, point symmetries, formula units, and experimental compounds, where available, are enumerated for each rotation pattern. The references for the experimental compounds can be found in reference 3 and are not be repeated here. "-" indicates no known example.

\begin{tabular}{|c|c|c|c|}
\hline Tilt & Space & Point & Known \\
\hline Systems & Group & Symmetry & Example \\
\hline 1. $a^{0} a^{0} a^{0}$ & $\operatorname{Pr} \overline{3} m(221) \mathrm{Z}=1$ & $m \overline{3} m\left(O_{h}\right)$ & $\mathrm{SrTiO}_{3}$ \\
\hline 2. $a^{0} a^{0} c^{+}$ & $P 4 / m b m(127) \mathrm{Z}=2$ & $4 / m m m\left(D_{4 h}\right)$ & $\begin{array}{c}\mathrm{CsSnI}_{3} \\
(351-425 \mathrm{~K}) \\
\end{array}$ \\
\hline 3. $a^{0} b^{+} b^{+}$ & $I 4 / m m m(139) \mathrm{Z}=8$ & $4 / m m m\left(D_{4 h}\right)$ & - \\
\hline 4. $a^{+} a^{+} a^{+}$ & $\operatorname{Im} \overline{3}(204) \mathrm{Z}=8$ & $m \overline{3}\left(T_{h}\right)$ & $\mathrm{Ca}_{0.25} \mathrm{Cu}_{0.75} \mathrm{MnO}_{3}$ \\
\hline 5. $a^{+} b^{+} c^{+}$ & $I / m m m(71) \mathrm{Z}=8$ & $m m m\left(D_{2 h}\right)$ & - \\
\hline 6. $a^{0} a^{0} c^{-}$ & $I 4 / m c m(140) \mathrm{Z}=4$ & $4 / m m m\left(D_{4 h}\right)$ & $\begin{array}{c}\mathrm{SrTiO}_{3} \\
(<110 \mathrm{~K})\end{array}$ \\
\hline 7. $a^{0} b^{-} b^{-}$ & $\operatorname{Imma}(74) \mathrm{Z}=4$ & $m m m\left(D_{2 h}\right)$ & $\begin{array}{c}\mathrm{PrAlO}_{3} \\
(151-205 \mathrm{~K})\end{array}$ \\
\hline 8. $a^{-} a^{-} a^{-}$ & $R \overline{3} c(167) \mathrm{Z}=4$ & $\overline{3} m\left(D_{3 h}\right)$ & $\mathrm{LaNiO}_{3}$ \\
\hline 9. $a^{0} b^{-} c^{-}$ & $C 2 / m(12) \mathrm{Z}=4$ & $2 / m\left(C_{2 h}\right)$ & $\begin{array}{c}\mathrm{PrAlO}_{3} \\
(<135 \mathrm{~K})\end{array}$ \\
\hline 10. $a^{-} b^{-} b^{-}$ & $C 2 / c(15) \mathrm{Z}=4$ & $2 / m\left(C_{2 h}\right)$ & - \\
\hline 11. $a^{-} b^{-} c^{-}$ & $P \overline{1}(2) \mathrm{Z}=2$ & $\overline{1}\left(C_{i}\right)$ & $\begin{array}{c}\mathrm{WO}_{3} \\
(230-300 \mathrm{~K})\end{array}$ \\
\hline 12. $a^{0} b^{+} c^{-}$ & Cmcm (63) $\mathrm{Z}=8$ & $m m m\left(D_{2 h}\right)$ & $\begin{array}{c}\mathrm{SrZrAlO}_{3} \\
(973-1103 \mathrm{~K}) \\
\end{array}$ \\
\hline 13. $a^{+} b^{-} b^{-}$ & Pnma (62) $\mathrm{Z}=4$ & $m m m\left(D_{2 h}\right)$ & $\mathrm{LaMnO}_{3}$ \\
\hline 14. $a^{+} b^{-} c^{-}$ & $P 2_{1} / m(11) \mathrm{Z}=4$ & $2 / m\left(C_{2 h}\right)$ & $\mathrm{GaLiBr}_{3}$ \\
\hline 15. $a^{+} a^{+} c^{-}$ & $P 4_{2} / n m c(137) \mathrm{Z}=8$ & $4 / m m m\left(D_{2 h}\right)$ & $\mathrm{CaFeTi}_{2} \mathrm{O}_{3}$ \\
\hline
\end{tabular}

the lattice vectors and the point symmetry operations, which are rotations, reflections, and inversions, or some combinations of these. Excluding the translations one obtains the subgroup of operations known as the point symmetry group. Although in principle objects can have many configurations and infinite number of point symmetry groups, the crystalline environment puts severe limitations on the allowed configurations and results in 32 crystallographic point groups.

Space Groups.-When a crystal is formed by inserting a motif into a Bravais lattice, the full symmetry of the crystal depends on both the symmetry of that object and that of the Bravais lattice; the full symmetry group is called the space group of the crystal. As a first order approximation, then, the total number of space groups can be simply compounded out of the 32 crystallographic point groups and the translation symmetry of the motif. There are additional considerations, however, in the case of the space groups that do not result from simple compounding of the Bravais lattice systems with the respective point groups. In the space group, a point is considered invariant if it is either left in place by a particular symmetry operation or is carried over to a position in an adjacent unit cell, which is reached by a simple translation of one unit cell. Also, the presence of different atomic motifs within the crystal and their different sets of symmetry operations mean that the rotational axes and the symmetry planes need not coincide at a common point. As a result more than one set of operations may fall within the same class. For these reasons, two additional types of compound symmetry operations appear in space groups: the screw axis and the glide planes are unique for the space groups. A screw axis operation produces a rotation followed by a translation along the axial direction while a glide plane operation yields reflection across a plane followed by translation along that plane. This results in total of 230 space groups. The Glazer systems for perovskites with octahedral rotations are found in 15 of the general 230 available space groups.

Site Symmetry.-Within each unit cell defined by a crystallographic space group, there are special sets of positions in which each point of the set has identical surroundings, because one or more symmetry elements coincide at that position. These points, or sites, and the set of symmetry operations that leave them invariant define the site group of the set of points; one point belonging to this set is called a Wyckoff position. The site group is a subgroup of the space group and is isomorphous with one of the 32 crystallographic point groups compatible with the space group. The number of equivalent points $n$ in a site group is equal to the order of the factor group (space group) $H$ divided by the order of the site group $h$, 
i.e., $n=H / h$. For example, in the simplest Glazer tilt system without any octahedral rotations, $a^{0} a^{0} a^{0}$ (space group $O_{h}^{1}$ or $P m \overline{3} m$ ), the $A$-site atom belongs to the site group $1 a$, with site symmetry $O_{h}$, the $B$-atom belongs to the site group $1 b$ with site symmetry $O_{h}$, and the three oxygen atoms belong to the site group $3 c$ with site symmetry $D_{4 h} \cdot{ }^{24}$ The importance of site symmetry becomes evident in the next section where we discuss the methods of normal mode determination in a crystal.

\section{NORMAL MODE DETERMINATION}

\section{A. General Approaches}

Of the various methods that are available to analyze the primitive unit cell and determine the selection rules for the first order phonon spectrum ${ }^{25-29}$ the Nuclear Site Analysis (NSA) method have been found to be most convenient since it requires the least amount of information about the unit cell; usually the Wyckoff positions and the site groups of the atoms are all that are required. Since for most practical purposes the $A B \mathrm{O}_{3}$ perovskites can be considered as a flexible network of corner connected $\mathrm{BO}_{6}$ octahedra, determining the Wyckoff positions and sites groups are relatively easy for these systems, and we use the NSA method in the present article.

To understand the approach in the NSA method, consider for example, a set of atoms with site group $g$ inside the unit cell of a crystal of space group $G$. If the order of $G$ is $H$ and that of $g$ is $h$, then $n=H / h$, where $n$ is the number of equivalent points (e.g., atoms) in the site group $g$. Since $g$ is a subgroup of $G$, each irrep of $g$ will map onto one or more of the irreps of $G$. In the NSA method only the vector irreps of the site group $g$ are chosen and these are then mapped onto the irreps of the crystallographic point group of the space group $G$. The total number of normal modes of a crystal with a particular space group can finally be obtained by considering all the site groups and occupied Wyckoff positions and taking their algebraic sum.

\section{B. Methodology for Perovskites with Octahedral Rotations}

We use the Nuclear Site Group Analysis (NSA) method to enumerate the normal modes of $A B \mathrm{O}_{3}$ perovskite oxides characterized by rotations and steric constraint driven distortions of the $B \mathrm{O}_{6}$ octahedra. We begin with the prototypical aristotype cubic polymorph, e.g., roomtemperature $\mathrm{SrTiO}_{3}$, with the symmetry $O_{h}^{1}(P m \overline{3} m)$. To generate the rotationally distorted phases, we then freeze-in each specific symmetry lowering rotation pattern (Table I, column 1) into the cubic structure to obtain the space groups in column 2 of Table I. The rotations lead to a splitting of the oxygen Wyckoff orbits, which have been tabulated elsewhere. ${ }^{30}$
TABLE II. Occupied Wyckoff positions and site symmetries for Pnma orthorhombic (space group $62, a=5.743, b=7.695$, $c=5.537 \AA) \mathrm{LaMnO}_{3}$ from Ref. 32 .

\begin{tabular}{lcclll}
\hline \hline & Wyckoff & \multirow{2}{*}{ Site } & \multicolumn{3}{c}{ Position } \\
\cline { 4 - 6 } Atoms & Orbit & Symmetries & $x$ & $y$ & $z$ \\
\hline $\mathrm{La}$ & $4 c$ & $C_{s}^{x z}$ & 0.55 & $\frac{1}{4}$ & 0.009 \\
$\mathrm{Mn}$ & $4 a$ & $C_{i}$ & 0 & 0 & 0 \\
$\mathrm{O}(1)$ & $4 c$ & $C_{s}^{x z}$ & -0.011 & $\frac{1}{4}$ & -0.071 \\
$\mathrm{O}(2)$ & $8 d$ & $C_{1}$ & 0.309 & 0.039 & 0.225 \\
\hline \hline
\end{tabular}

Knowledge of the occupied cation and anion Wyckoff positions in the standard notations is essential to the NSA method, because they are directly related to the site symmetry of the atomic species. The normal modes of each atom are identified by consulting the International Tables for Crystallography (ITC) and/or Porto et al. ${ }^{29,31}$ for the character tables of the site groups and of the space group. These elements are required for determining the vector irreps of the site group and subsequently mapping these to the irreps of the space group. We repeated this analysis for all atomic sets until the total number of normal modes for the asymmetric unit was obtained. The data are presented in Tables III - XVII found in the appendix.

\section{Example: Normal Mode Determination of the $a^{+} b^{-} b^{-}$Rotation Pattern}

We now describe this process in detail for the orthorhombic $a^{+} b^{-} b^{-}$rotation pattern, Glazer tilt system 13 (Table XV), which $\mathrm{LaMnO}_{3}$ is known to exhibit. ${ }^{32}$ When the tilt system $a^{+} b^{-} b^{-}$is applied to the cubic structure the space group transforms from cubic $P m \overline{3} m$ to orthorhombic Pnma. The displacement pattern of oxygen atoms also leads to an approximate $\sqrt{2} \times \sqrt{2} \times 2$ increase in the cell dimensions $a, b$ and $c$, respectively, producing a unit cell with four $A B \mathrm{O}_{3}$ formula units $(Z=4)$, as enumerated in column 2 of Table I. In Schoenflies notation, the Pnma space group is equivalent to $D_{2 h}^{16}$, making the relevant point symmetry $m m m$ or $D_{2 h}$ (Table XV).

After obtaining the lower symmetry structure (Table II), the Wyckoff positions can be read from the ITC, or by using crystallographic visualization software such as VESTA and Crystalmaker. ${ }^{31,33}$ Note that in the Wyckoff orbit column, the numeral preceding the Wyckoff label indicates that all of those symmetry equivalent atoms occupy that particular Wyckoff position. Chemically equivalent atoms can occupy crystallographically unique Wyckoff positions in crystals. This becomes apparent in $\mathrm{LaMnO}_{3}$ with the $a^{+} b^{-} b^{-}$rotation pattern: The eight oxygen atoms found in the $a c$ plane occupy Wyckoff positions $8 d$ and possess site symmetry $C_{1}$, whereas the four oxygen atoms along the $b$ axis occupy Wyckoff positions $4 c$ and possess site symmetry $C_{s}^{x z}$ (Table II). This "splitting" 
of the Wyckoff orbits of the $\mathrm{O}$ atoms is a signature of the structural transition on going from $P m \overline{3} m \rightarrow P n m a$, which can be exploited in Raman spectroscopy studies to recognize the onset of the transition by monitoring the change in number of peaks (modes), intensities, etc., with external stimuli. In the ideal cubic case, however, only one Wyckoff position is necessary to describe all oxygen atoms in the crystal structure.

For determining the normal modes, consider the four oxygen atoms occupying the Wyckoff position $4 c$ and site symmetry $C_{s}$, the irreps of which can be found in the ITXRC and/or Porto et al.. ${ }^{29,31}$ The vector irreps of $C_{s}$ are $A^{\prime}$ and $A^{\prime \prime}$. When these are mapped on to the irreps of the crystallographic point group $D_{2 h}$ of Pnma, we obtain the contributions of these four oxygen atoms to the normal modes of $\mathrm{LaMnO}_{3}$. This is repeated for all the atomic species in the unit cell.

The first column of Table XV shows all available normal modes for the tilt system $a^{+} b^{-} b^{-}$in the absence of any other atomic distortions (cation displacements or JahnTeller distortions of the ligands). The values in the main body of the table shows the number of symmetry modes in which the atoms participate. This type of presentation, instead of simply writing down all the modes, provides physical meaning to the modes. As shown in Table XV, the 4 La atoms have site-symmetry $C_{s}^{x z}$ and contribute the following modes:

$$
2 A_{g} \oplus A_{u} \oplus B_{1 g} \oplus 2 B_{1 u} \oplus 2 B_{2 g} \oplus B_{2 u} \oplus B_{3 g} \oplus 2 B_{3 u} .
$$

The $4 \mathrm{Mn}$ atoms with site-symmetry $C_{i}$ contribute:

$$
3 A_{u} \oplus 3 B_{1 u} \oplus 3 B_{2 u} \oplus 3 B_{3 u},
$$

while the $4 \mathrm{O}(1)$ atoms with site-symmetry $C_{s}^{x z}$ contribute:

$$
2 A_{g} \oplus A_{u} \oplus B_{1 g} \oplus 2 B_{1 u} \oplus 2 B_{2 g} \oplus B_{2 u} \oplus B_{3 g} \oplus 2 B_{3 u},
$$

and the $8 \mathrm{O}(2)$ atoms with site symmetry $C_{1}$ contribute:

$$
3 A_{g} \oplus 3 A_{u} \oplus 3 B_{1 g} \oplus 3 B_{1 u} \oplus 3 B_{2 g} \oplus 3 B_{2 u} \oplus 3 B_{3 g} \oplus 3 B_{3 u} .
$$

The last two columns in Table XV provide the mode selection rules in standard notations. The acoustic modes belong to representations which contain the translation vectors, symbolized by $T$. After subtracting out the three acoustic modes available to three-dimensional crystals, the remaining modes labeled by $T$ are IR active. The selection rules for the Raman modes are shown separately in the last column with the polarizability tensor components, denoted by $\alpha_{i j}$ and the appropriate Cartesian subscripts. $\alpha_{i j}$ are also known as susceptibility derivative tensors in the literature.

For Pnma $\mathrm{LaMnO}_{3}$, Table XV reveals that one of each of the $B_{1 u}, B_{2 u}$ and $B_{3 u}$ modes account for the acoustic (translational) modes of the crystal. The remaining $9 B_{1 u}$, $3 B_{2 u}$ and $8 B_{3 u}$ modes are IR-active, and the $7 A_{g}, 5 B_{1 g}$, $7 B_{2 g}$, and $5 B_{3 g}$ modes are Raman active. The normal modes that are neither IR active, nor Raman active are considered silent. In the present case, the silent modes transform with $A_{u}$ symmetry.

For completeness, we also provide the rotation (axial) vectors compatible with the appropriate modes. For example, as shown in Table $\mathrm{XV}$, the rotation $R_{z}$ has the same symmetry properties as that of the $B_{1 g}$ modes. In general, the rotations have the same symmetry properties as those of the difference between anti-symmetric Raman tensors: $R_{z}$ transforms as $\alpha_{x y}-\alpha_{y x}$. For Raman scattering that uses excitation frequency far from electronic resonance, these differences are zero since the polarizability tensors are symmetric. In resonance Raman experiments, however, asymmetries in the polarizability tensors are introduced and then such modes, i.e., modes with $R_{x}, R_{y}, R_{z}$ labels could become Raman active.

The last row of each table shows the total number of modes for each rotation pattern. Note that modes with $A$ and $B$ spectroscopic labels are non-degenerate, whereas modes with $E(T)$ labels are doubly (triply) degenerate.

Finally, to complement these tables we have developed a graphical description of each mode by using the character projection method. The complete compilation of all 705 of these diagrams is available by accessing: http://meso.materials.drexel.edu/Glazer_modes. A few of the representative diagrams are shown in Figure 2. The vector displacements without such diagrams, corresponding to all normal modes for each Wyckoff position available to the 230 space groups, can also be obtained using the Bilbao Crystallographic Server and the web-based program SAM.

\section{DISCUSSIONS AND APPLICATIONS}

\section{A. Raman Mode Bookkeeping: How many modes are there?}

One way to check the accuracy of the normal mode tables we have generated for the $A B \mathrm{O}_{3}$ perovskites with octahedral rotations (Tables III-XVII) is to count the number of normal modes enumerated for each crystal system. If there are $p>2$ different atoms in a threedimensional primitive cell, there should be $3 p$ branches to the phonon dispersion relation, and consequently, $3 p$ modes at the $\Gamma$ point. Of these $3 p$ modes, 3 are acoustic (one longitudinal and two transverse) modes and the remaining, $3 p-3$, are optical modes. For space groups with a primitive lattice, as is the case for the Glazer tilt systems 1, 2, 8, 11, 13, 14, and 15, the Raman mode enumeration is straightforward.

For the Glazer systems with non-primitive lattice, care must be taken to avoid redundancy in the enumeration of the normal modes. For these systems the usual construction of the primitive cell is less useful because it doesn't usually reveal the full symmetry of the structure. In these cases the crystallographic cell or the conventional cell is constructed by tiling an integral number of primitive cells through lattice translations of the primitive cell 


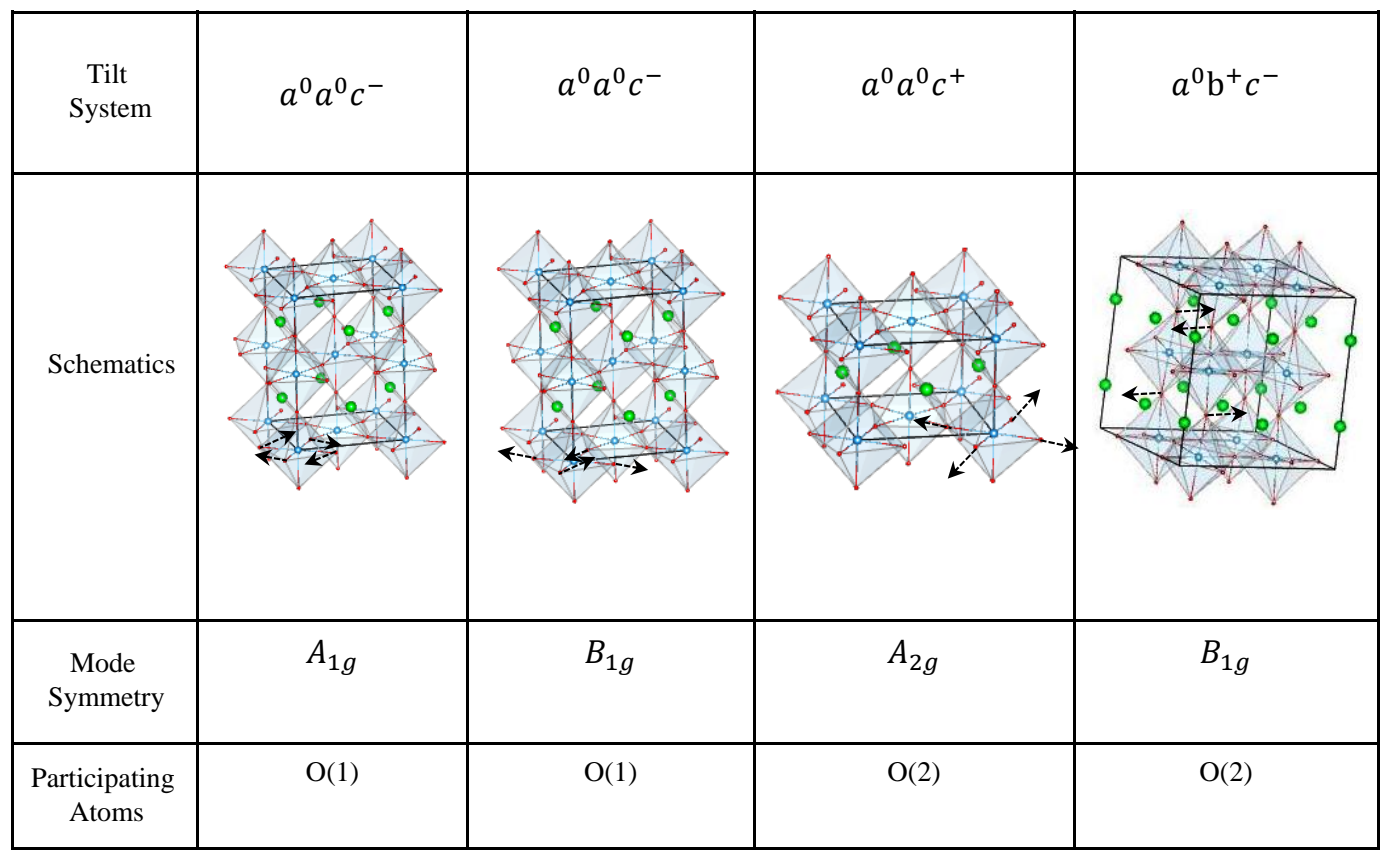

FIG. 2. (Color online) Schematic atomic vibrational patterns of selected normal modes for a few octahedral rotation patterns. The symmetry mode symbols (irreducible representations) and the atoms participating in the normal modes are also provided. The complete database for all normal modes in each Glazer tilt system can be viewed online by accessing: http://meso.materials.drexel.edu/Glazer_modes.

lattice point. Since the translation group transforms as the identity element of the space group, the distribution of degrees of freedom among the space group irreps does not change. However, this introduces a redundancy in the value of the characters for each symmetry: Each set of equipoints in the primitive cell is duplicated for the additional lattice points in the expanded crystallographic cell. Since redefining the unit cell vectors does not affect the rotational symmetry, the redundancy is uniformly distributed over the characters of all symmetry classes of the space group.

To recover the primitive $(\mathrm{P})$ cell characters, one should divide the conventional $(\mathrm{C})$ cell characters by the lattice point multiplicity, i.e., $\chi_{\mathrm{P}}=\chi_{\mathrm{C}} / \ell$, where $\ell$ is the number of lattice points (or primitive cells) in the full crystallographic cell: $\ell=4$ for face-centered cell, $\ell=3$ for rhombohedral $R$-centered cells given in hexagonal settings, and $\ell=2$ for both body centered cells and base centered cells. ${ }^{26}$ This consequently yields the number of normal modes being an integral fraction of $3 q$, where $q$ is the number of atoms in the conventional cell. The Glazer systems $3,4,5,6$, and 7 are body centered and as such the number of modes is $3 q / 2$. The Glazer system 8 is rhombohedral and as such the number of modes is $3 q / 3$. The Glazer systems 9,10 and 12 are base centered and as such the number of modes is $3 q / 2$.

\section{B. Applications: Phase Transitions in Bulk Perovskites}

Having ensured that the number of modes for each system is accurate, we now provide some illustrative applications and discuss a few limitations of the constructed tables and the schematics. Here we show how the Raman normal modes determination of the various octahedral rotation patterns in perovskites provides a manner to explore antiferrodistortive phase transitions.

$\mathrm{CaTiO}_{3}$ is a widely used electroceramic and has been recognized as a suitable material for the immobilization of high-level radioactive wastes. ${ }^{34}$ Consequently, the properties of $\mathrm{CaTiO}_{3}$, especially its crystal structures over different temperature regimes have lately been the focus of intense studies. ${ }^{35}$ It undergoes a series of structural transitions driven by the octahedral rotations (Figure 3): ${ }^{36}$ Under ambient conditions, $\mathrm{CaTiO}_{3}$ is orthorhombic (space group Pnma) and exhibits the $a^{+} b^{-} b^{-}$rotation pattern. Upon heating, the structure transforms to tetragonal (space group $I 4 / \mathrm{mcm}$, Glazer system $a^{0} a^{0} c^{-}$) at $1520 \mathrm{~K}$. Note that some studies suggest that between the tetragonal and the orthorhombic phase there is an additional structure with orthorhombic symmetry, $\mathrm{Cmcm}$, that exhibits one in-phase and one out-of-phase rotation $\left(a^{0} b^{+} c^{-}\right)$. Finally near $1645 \mathrm{~K}, \mathrm{CaTiO}_{3}$ transforms into the ideal cubic $P m \overline{3} m$ structure without any octahedral rotations $\left(a^{0} a^{0} a^{0}\right)$.

Unpolarized Raman studies enable identification of 


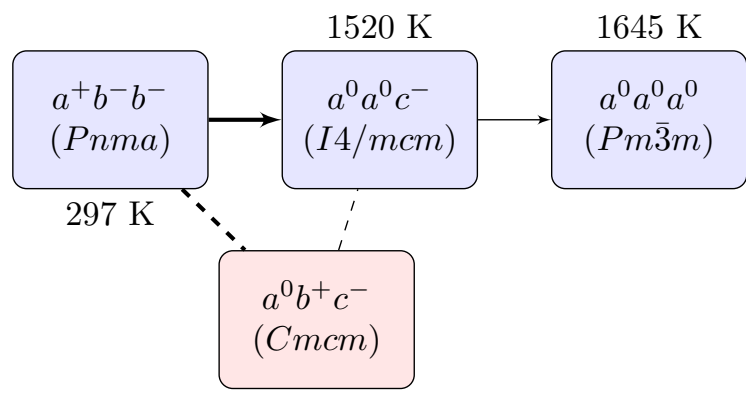

FIG. 3. Sequence of structural phase transitions and the change in octahedral rotation patterns that occurs with increasing temperature in perovskite $\mathrm{CaTiO}_{3}$. The orthorhombic $\mathrm{Cmcm}$ phase (linked by broken lines) appears (or is sometimes absent) experimentally as an intermediate phase between $P n m a$ and $I 4 / \mathrm{mcm}$ at intermediate temperatures. Transitions required by Landau theory to be first (second) order are distinguished by heavy (light) lines.

the nature and onset of the phase transition from the octahedrally rotated structures to the cubic structure upon heating. The relevant information for the analysis of the rotation-driven structural transitions

$$
a^{+} b^{-} b^{-} \rightarrow a^{0} b^{+} c^{-} \rightarrow a^{0} a^{0} c^{-} \rightarrow a^{0} a^{0} a^{0}
$$

are found in Tables XV, XIV, VIII and III, respectively. First, comparing these tables reveals that all of the rotationally distorted perovskite polymorphs have first-order Raman active vibrational modes, whereas all zone-center modes in cubic $\left(a^{0} a^{0} a^{0}\right)$ structure are Raman inactive. Accordingly, unpolarized Raman spectra of the tilted structures should exhibit first-order Raman features, possibly superimposed on broad second order features, where as the cubic should not. (Note that in practice, however, at the transition to the cubic structure the first order Raman features could give way to broad second order features - such effects are seen in prototypical cubic perovskite $\mathrm{SrTiO}_{3} \cdot{ }^{35}$ )

Polarized Raman studies can be used to determine the phase transitions among the tilted structures. Raman scattering cross sections $\left(\sigma_{R}\right)$ for Stokes scattering is related to the polarizations of the incident $\left(\epsilon_{I}^{i}\right)$ and scattered $\left(\epsilon_{S}^{j}\right)$ radiations and the susceptibility (derivative) tensors by the following formula:

$$
\sigma_{R} \propto\left|e_{S}^{i} e_{I}^{j} \alpha_{i j}\right|^{2},
$$

where $\alpha_{i j}$ are the components of the susceptibility (derivative) tensors appropriate for a given mode, shown in the last column of the Tables III-XVII. Note that summation over the repeated cartesian indices $(i, j)$ is implicit in the above equation.

As an application of this, let's consider Raman spectroscopy in the back scattering geometry for $\mathrm{CaTiO}_{3}$. The standard scattering geometry for this experiment is $z(i j) z$, which is shorthand for $k_{I}\left(\mathrm{e}_{I}^{i}, \mathrm{e}_{S}^{j}\right) k_{S}$, where $k_{I}$ and $k_{S}$ are the wave vectors of the incident and scattered light, respectively, and $\mathrm{e}_{I}^{i}$ and $\mathrm{e}_{S}^{j}$ are their corresponding polarization vectors. Since both $k_{S}$ and $k_{I}$ are in the $z$ direction, the polarization vectors of both the incident and scattered radiation are in the $x y$-plane, resulting in Raman cross section of:

$$
\sigma_{R} \propto\left|e_{S}^{x} e_{I}^{x} \alpha_{x x}+e_{S}^{x} e_{I}^{y} \alpha_{x y}+e_{S}^{y} e_{I}^{x} \alpha_{y x}+e_{S}^{y} e_{I}^{y} \alpha_{y y}\right|^{2} .
$$

Consider now the transition from $a^{+} b^{-} b^{-} \rightarrow a^{0} a^{0} c^{-}$. As seen from Tables XV and VIII, there are significant differences between the mode distributions in the two structures. In the $a^{+} b^{-} b^{-}$(Pnma) structure, the Raman active modes are:

$$
7 A_{g} \oplus 5 B_{1 g} \oplus 7 B_{2 g} \oplus 5 B_{3 g},
$$

whereas in the $a^{0} a^{0} c^{-}(I 4 / m c m)$ system, the Raman active modes are:

$$
A_{g} \oplus B_{g} \oplus 2 B_{2 g} \oplus E_{g} .
$$

Based on the larger number of Raman modes in Pnma (24 compared to the 8 in $I 4 / m c m \mathrm{CaTiO}_{3}$ ), there should be a readily detectable difference in the Raman spectra, i.e., one should expect significantly more first-order Raman features in the Pnma structure than in $I 4 / \mathrm{mcm}$, even in unpolarized Raman spectroscopy. The two phases can further be distinguished by performing a careful polarized Raman experiment, whereby an appropriate selection of the incident and scattered light orientation will enhance or suppress a first-order Raman mode. Raman spectroscopy in the $z(x x) z$ geometry will result in Raman cross sections of

$$
\sigma_{R} \propto\left|e_{S}^{x} e_{I}^{x} \alpha_{x x}\right|^{2} .
$$

This will only show modes of $A_{g}$ symmetry of which are seven in the Pnma and only one in the $I 4 / \mathrm{mcm}$ structure. Similar results can be expected in the $z(y y) z$ geometry. Further careful studies with the $z(y z) y$ configuration would show first order Raman features from the singly degenerate $B_{3 g}$ mode in Pnma $\mathrm{CaTiO}_{3}$, whereas an $E_{g}$ doublet would appear in the $I 4 / \mathrm{mcm}$ structure.

The controversial phase transition from Pnma to $\mathrm{Cmcm}$ would be more difficult to determine by examining the Raman spectra alone, since the Raman active modes present in the two structures are similar (cf. Tables $\mathrm{XV}$ and XIV). To remedy the ambiguity, one could rather use the octahedral rotation angle, specifically the component about the $x$-axis, as an order parameter for the $P n m a \rightarrow C m c m$ transition, because it goes from a finite to null value as the rotation pattern changes, i.e. $a^{+} b^{-} b^{-}$ to $a^{0} b^{+} c^{-}$. Recently, Dubroka et al. and Abrashev et al. have shown how to correlate the Raman $A_{g}$ mode frequency with the tilt angle, and subsequently that tilt angle with the free oxygen positions. ${ }^{14,16}$ Their results, along with our schematic diagrams for the normal modes of the rotationally distorted perovskites and lattice dynamic calculations could be used to ascertain the phase transition between these two structures. ${ }^{36,37}$ 


\section{Applications: Structure-property Relations in Perovskite Thin Films and Superlattices}

We expect this methodology to have higher impact in $A B \mathrm{O}_{3}$ perovskite systems realized via octahedral engineering route whereby efforts are being made to exploit epitaxial strains, interfacial octahedral coupling, and superlattices to stabilize structures inaccessible via conventional solid-state chemistry techniques. Recent computational studies predict selective control of the octahedra about different crystal directions by using epitaxial strain to control the rotation-lattice coupling in epitaxial films. ${ }^{38,39}$ Strain can therefore be used to directly tailor the flavor magnitude and phase - of octahedral rotation patterns in thin films.

The normal mode determination presented here, could be used to design and analyze $A B \mathrm{O}_{3}$ films with octahedral rotations. Consider for example, $\mathrm{La}_{1 / 3} \mathrm{Sr}_{2 / 3} \mathrm{FeO}_{3}$ (LSFO), which is rhombohedral (Glazer tilt system $a^{-} a^{-} a^{-}$) in the bulk. Its normal modes appear in Table X. However, if an epitaxial film of LSFO is grown on cubic $\mathrm{SrTiO}_{3}$, it would likely transform to a monoclinic phase with the $a^{-} b^{-} b^{-}$octahedral rotation pattern due to lattice mismatch between the film and the substrate in the epitaxial plane. Reduction of symmetry will lead to a splitting of some degenerate modes of the rhombohedral system, which can be determined by using the standard reduction formulae $^{40}$ and our Table XII, i.e., the relevant normal modes for the monoclinic LSFO. By comparing the Raman spectra of bulk LSFO and the epitaxial film, it should be possible to evaluate the change in crystal structure for the rhombohedral to monoclinic transition.

Formation of a coherent perovskite-perovskite heterointerface produces a geometrical constraint of the octahedral connectivity across the interface in synthetic structures. ${ }^{6}$ The octahedral rotations present in a substrate, for example, can propagate into the near-interface region of a thin film: This was recently observed experimentally using high-resolution x-ray diffraction and synchrotron radiation, in $\left(\mathrm{LaNiO}_{3}\right)_{n} /\left(\mathrm{SrMnO}_{3}\right)_{2}$ superlattices. ${ }^{41}$ This so-called octahedral proximity effect remains to be harnessed for materials design, yet has the ability to mediate electron-lattice coupling across interfaces and produce remarkable changes in the electronic properties of ultrathin films and superlattices. Segal et al. have shown that the propagation of lattice vibrational modes (phonons) associated with the dynamic octahedral rotations causes drastic changes in the resistivity of a $\mathrm{La}_{0.53} \mathrm{Sr}_{0.47} \mathrm{MnO}_{3}$ (LSMO) films as the system is cooled through the $a^{0} a^{0} a^{0} \rightarrow a^{0} a^{0} c^{-}$phase transition of the $\mathrm{SrTiO}_{3}$ substrate. The proposed microscopic mechanism was attributed to evanescent cross-interface coupling between the charge carriers in the film and the soft phonon mode responsible for the out-of-phase rotation of $\mathrm{TiO}_{6}$ in $\mathrm{SrTiO}_{3}$. In other words, enhanced electron-lattice coupling mediated through the correlated oxygen octahedral motions. ${ }^{42}$

Other external stimuli besides temperature can pro- mote novel behavior mediated by octahedral rotations. For example, Caviglia et al. have shown that an optical excitation in resonance with a stretching mode of a perovskite substrate can trigger a dynamic (transient) insulator to metal transition in $\mathrm{NdNiO}_{3}$ epitaxial films below the bulk Néel temperature. ${ }^{43}$ Recent Raman spectroscopy studies on superlattices of superconducting $\mathrm{YBa}_{2} \mathrm{Cu}_{3} \mathrm{O}_{7}$ and magnetoresistive $\mathrm{La}_{2 / 3} \mathrm{Sr}_{1 / 3} \mathrm{MnO}_{3}$ below the critical temperature of the cuprate, reveal that the superconducting state can renormalize the lineshape of the $\mathrm{MnO}_{6}$ octahedral rotation modes in the manganite, providing an unexplored platform to tailor electron-lattice interactions in correlated materials. ${ }^{44}$

The unique effects described here are all provided by the normal mode distributions within the materials and their coupling to the low-energy electronic structure. They could possess tremendous technological potential, yet the microscopic mechanisms in nearly all cases remain inadequately understood and characterized. Much of the current results of computational studies, for example, await experimental verifications. Segal et al. suggested that the change in conductivity in their LSMO epitaxial film could be due to the alteration of the $B-\mathrm{O}-B$ bond angles in the film by the soft phonon modes of the STO substrate - direct measurement of these variations remains to be seen. Note however that they also offered an equally likely scenario: The substrate could modify the $B-\mathrm{O}$ bond lengths in the film, resulting in similar modifications to the films conductivity, via a bond stretching mode. In the experiments by Caviglia et al., the authors acknowledge that the precise nature of the interaction between the LAO substrate and the $\mathrm{NdNiO}_{3}$ film remains poorly understood and merits systematic studies. For that reason, the group offered an alternative explanation for the dynamic insulator-metal transition: The stretching mode of the substrate could couple to a rotational mode (soft mode) of the film, which would also alter the films conductivity through a modification of the Ni $3 d-$ O $2 p$ angular orbital overlap.

For such complex systems, our results presented here, especially the normal mode schematics, could be useful in drawing more decisive conclusions. By superimposing a unit cell of an epitaxial material on a unit cell of the intended substrate and inducing a particular normal mode in the substrate, once could examine geometrically and on symmetry grounds the extent to which the substrate modes couple to the film and induce changes in both the topology of the film's perovskite lattice and properties. Since our paper presents a complete list of normal modes, selection rules, and schematic diagrams of the bulk $A B \mathrm{O}_{3}$ perovskites with octahedral rotations, careful implementation of our results could guide research avenues in search of quantum phase transitions in artificial materials using optical excitation of lattice modes. 


\section{Applications: Normal Mode Determination of a Non-Glazer System}

As an extension of the methodology presented here, we describe the method for normal mode determination of a non-Glazer system that undergoes rotation of the $\mathrm{BO}_{6}$ octahedra as well as off-centering displacements of the ions: $\mathrm{BiFeO}_{3}$ is a magnetoelectric multiferroic whose ground state exhibits both antiferromagnetic and ferroelectric order. Bulk $\mathrm{BiFeO}_{3}$ has the rhombohedral $R 3 c$ structure, which consists of antiferrodistortive octahedral rotation $\left(a^{-} a^{-} a^{-}\right)$around the [111] direction and an additional relative off-centering of anions and cations along the [111] direction leading to a ferroelectric polarization around that axis. Using method similar to $\mathrm{LaMnO}_{3}$ (Sec. IV C) we construct the normal mode table for $\mathrm{BiFeO}_{3}$ in the bulk (Table XVIII).

These bulk normal modes, however, should change when an epitaxial film of $\mathrm{BiFeO}_{3}$ is grown under epitaxial strain on a substrate with different symmetry. If rhombohedral $\mathrm{BiFeO}_{3}$ is deposited on a cubic (001) substrate like STO, the film, under biaxial strain, will acquire monoclinic symmetry due to the geometric constraints imposed by epitaxy. In this case the Glazer notation of this film becomes $a^{-} b^{-} b^{-}$and the relevant normal mode table to use is Table XII. If the substrate is cubic but terminated with a (110) surface, rhombohedral (001) or orthorhombic (001) the epitaxial film would adopt a triclinic structure. ${ }^{6}$ Using the appropriate tables and the selection rules, a polarized Raman experiment would be able to determine the mode and nature of substrate induced strain effects on the $\mathrm{BiFeO}_{3}$ epitaxial films.

\section{E. Limitations}

We believe we would be remiss if we omitted a discussion of some limitations of the current paper and the methods used to derive the normal mode distributions. The normal modes in our paper were derived for simple, single phase, $A B \mathrm{O}_{3}$ perovskites with octahedral rotations. In mixed $A$ - and $B$-site systems, e.g. $\mathrm{La}_{1-y} \mathrm{Sr}_{y} \mathrm{Mn}_{1-x} M_{x} \mathrm{FeO}_{3}(M$ $=\mathrm{Cr}, \mathrm{Co}, \mathrm{Cu}, \mathrm{Zn}, \mathrm{Sc}$, or $\mathrm{Ga}$ ), the normal modes may be drastically different from their simple $A B \mathrm{O}_{3}$ counterpart. ${ }^{16}$ In these cases the $A$ and $B$ site disorder suppresses the translational invariance that is a requirement for the range of applicability of the symmetry analysis. The result being that the lineshapes of the modes with atomic displacement patterns from those sites could be broadened. Similar broadening could occur even in single phase perovskites grown in thin film form, where unintentional disorder and variability in the site occupation is induced preferentially during growth along various directions, i.e., in the plane of or perpendicular to the substrate. Such broadening can further lead to linewidths in which distinct modes of different, but closely spaced, energies are indistinguishable.

Second, our results were derived for bulk perovskites.
Their direct application to epitaxial films and superlattices may not be immediately transferable. As mentioned earlier, the Glazer octahedral tilt patterns are derived by considering the octahedra as rigid units. Even though we allowed for the distortions of the $\mathrm{BO}_{6}$ octahedra to account for steric constraints, in epitaxial films and superlattices the octahedra are necessarily further distorted. A consequence of such geometrically required distortions is that they can produce crystal structures with space groups different than those analyzed here, i.e., those obtained from Glazer's analysis of possible combinations of in- and out-of-phase rotations. Nevertheless, our schemes would be a good starting point in analyzing these systems and deviations from ideality. For example, a comparison based on the Raman mode predictions produced with our tables to the actual experimental results could help in understanding the perturbative nature of octahedral engineering in artificial perovskites.

Lastly, our treatment omits the consequences of magnetic ordering on the crystal symmetry, which is expected to become critical in perovskite superlattices formed with transition metal $B$-sites with open $d$-shell configurations. Several $\mathrm{ABO}_{3}$ type perovskites undergo paramagnetic metal to antiferromagnetic insulator transitions followed by charge ordering as they are cooled through their Néel temperature. The crystal symmetry operations in the antiferromagnetic state must leave invariant not only the positions of the ions but also their magnetic moments. For example, magnetic ordering in the rutile structure reduces the space group symmetry from that of rutile with non-magnetic cations $\left(P 4_{2} / m n m\right)$ to Pnnm or $D_{2 h}^{12},{ }^{45}$ which in turn alters the Raman mode distribution. We hope our paper spawns additional developments in the experimental Raman studies of artificial perovskite oxides, theoretical approaches to predict the normal mode distribution in such materials, and means to overcome the current limitations discussed.

\section{CONCLUSIONS}

Using the nuclear site group analysis method we have determined the complete set of normal modes and the associated selection rules for all 15 of the $A B X_{3}$ perovskites systems with octahedral rotations (the Glazer systems). For each mode, we have produced a corresponding schematic diagram showing the vector displacement pattern of the atoms participating in the particular mode. The results of this analysis is a compendium of 705 schematic diagrams, which are now web accessible.

We have shown how some recent experimental findings can be analyzed using our tables and the schematic diagrams to understand macroscopic interactions in the complex systems. We expect these tables and the schematic diagrams to be useful tools in analyzing the phase transitions, electron-lattice coupling and elucidating structureproperty relationships in $A B \mathrm{O}_{3}$ perovskites with octahedral rotations. Equipped with this database for the 
bulk perovskites, we suggest that one would be able to both analyze and rationally design functional artificial materials built out of these perovskite blocks and rotated octahedral units.

\section{ACKNOWLEDGMENTS}

Work supported by the ONR (N00014-11-1-0664). J.M.R. was supported by ARO (W911NF-12-1-0133). J.E.S. acknowledges support from the ARO (W911NF08-1-0067). Valuable discussions with Steven J. May are thankfully acknowledged. The authors are also grateful to Pierre-Eymeric Janolin for helpful comments.
* email: spanier@drexel.edu

1 D. G. Schlom, L.-Q. Chen, X. Pan, A. Schmehl, and M. A. Zurbuchen, Journal of the American Ceramic Society 91, 2429 (2008).

2 P. M. Woodward, Acta Crystallographica Section B 53, 32 (1997).

3 P. M. Woodward, Acta Crystallographica Section B 53, 44 (1997).

4 M. Medarde, J. Mesot, P. Lacorre, S. Rosenkranz, P. Fischer, and K. Gobrecht, Phys. Rev. B 52, 9248 (1995).

5 J.-S. Zhou, J. B. Goodenough, B. Dabrowski, P. W. Klamut, and Z. Bukowski, Phys. Rev. Lett. 84, 526 (2000).

6 J. M. Rondinelli, S. J. May, and J. W. Freeland, MRS Bulletin 37, 261 (2012).

7 P. Zubko, S. Gariglio, M. Gabay, P. Ghosez, and J.-M. Triscone, Annual Review of Condensed Matter Physics 2, 141 (2011), http://www.annualreviews.org/doi/pdf/10.1146/annurevconmatphys-062910-140445.

8 S. J. May, J.-W. Kim, J. M. Rondinelli, E. Karapetrova, N. A. Spaldin, A. Bhattacharya, and P. J. Ryan, Phys. Rev. B 82, 014110 (2010).

9 J. Hwang, J. Y. Zhang, J. Son, and S. Stemmer, Applied Physics Letters 100, 191909 (2012).

10 H. M. Christen, M. Varela, and D. H. Kim, Phase Transitions 81, 717 (2008).

11 D. A. Tenne and X. Xi, Journal of the American Ceramic Society 91, 1820 (2008).

12 M. DiDomenico, S. H. Wemple, S. P. S. Porto, and R. P. Bauman, Phys. Rev. 174, 522 (1968).

13 J. F. Scott, Phys. Rev. 183, 823 (1969).

14 M. V. Abrashev, A. P. Litvinchuk, M. N. Iliev, R. L. Meng, V. N. Popov, V. G. Ivanov, R. A. Chakalov, and C. Thomsen, Phys. Rev. B 59, 4146 (1999).

15 S. Ghosh, N. Kamaraju, M. Seto, A. Fujimori, Y. Takeda, S. Ishiwata, S. Kawasaki, M. Azuma, M. Takano, and A. K. Sood, Phys. Rev. B 71, 245110 (2005).

16 A. Dubroka, J. Humlíček, M. V. Abrashev, Z. V. Popović, F. Sapiña, and A. Cantarero, Phys. Rev. B 73, 224401 (2006).

17 M. N. Iliev and M. V. Abrashev, Journal of Raman Spectroscopy 32, 805 (2001).

18 T. Wolfram and S. Ellialtioglu, Electronic and Optical Properties of $d$-Band Perovskites (Cambridge University Press, 2006).

19 C. J. Howard and H. T. Stokes, Acta Crystallographica Section B 54, 782 (1998).

20 A. M. Glazer, Acta Crystallographica Section B 28, 3384 (1972).

21 A. M. Glazer, Acta Crystallographica Section A 31, 756 (1975).
22 A. Glazer, Phase Transitions 84, 405 (2011).

23 E. Salje, Acta Crystallographica Section A 32, 233 (1976).

24 An equivalent setting for the $a^{0} a^{0} a^{0}$ rotation pattern, has the $A$-site at Wyckoff position $1 b$, the B-site at $1 a$ and the oxygen atom at $3 d$.

25 S. Bhagavantum and T. Venkatarayudu, Proc. Indian Acad. Sci. Sect. A A9, 224 (1939).

26 B. A. DeAngelis, R. E. Newham, and W. B. White, Amer. Mineral. 57, 255 (1972).

27 R. S. Halford, J. Chem. Phys. 14, 8 (1946).

28 J. P. Mathieu, Spectres de Vibration et Symetrie des Molecules et des Cristaux (Herman et cie., Paris, 1945).

29 D. L. Rousseau, R. P. Bauman, and S. P. S. Porto, Journal of Raman Spectroscopy 10, 253 (1981).

${ }^{30}$ K. Knight, Canadian. Mineral. 47, 381 (2009).

31 International Union of Crystallography, in International Tables for Crystallography, Vol. A: Space Group Symmetry, edited by T. Hahn (Springer, Dordrecht, 2005).

32 J. B. Elemans, B. V. Laar, K. V. D. Veen, and B. Loopstra, Journal of Solid State Chemistry 3, 238 (1971).

${ }^{33}$ K. Momma and F. Izumi, Journal of Applied Crystallography 44, 1272 (2011).

34 A. E. Ringwood, S. E. Kesson, K. D. Reeve, D. M. Levins, and E. J. Ramm, Radioactive Waste Froms for the Future, edited by W. Lutze and R. C. Ewing (Elsevier, Amsterdam, 1988).

35 P. McMillan and N. Ross, Physics and Chemistry of Minerals 16, 21 (1988), 10.1007/BF00201326.

36 M. Yashima and R. Ali, Solid State Ionics 180, 120 (2009).

37 G. Gou, I. Grinberg, A. M. Rappe, and J. M. Rondinelli, Phys. Rev. B 84, 144101 (2011).

38 A. T. Zayak, X. Huang, J. B. Neaton, and K. M. Rabe, Phys. Rev. B 74, 094104 (2006).

39 A. J. Hatt and N. A. Spaldin, Phys. Rev. B 82, 195402 (2010).

40 B. Tsukerblat, Group Theory in Chemistry and Spectroscopy (Dover, New York, 2006).

41 S. J. May, C. R. Smith, J.-W. Kim, E. Karapetrova, A. Bhattacharya, and P. J. Ryan, Phys. Rev. B 83, 153411 (2011).

42 Y. Segal, K. F. Garrity, C. A. F. Vaz, J. D. Hoffman, F. J. Walker, S. Ismail-Beigi, and C. H. Ahn, Phys. Rev. Lett. 107, 105501 (2011).

43 A. D. Caviglia, R. Scherwitzl, P. Popovich, W. Hu, H. Bromberger, R. Singla, M. Mitrano, M. C. Hoffmann, S. Kaiser, P. Zubko, S. Gariglio, J.-M. Triscone, M. Först, and A. Cavalleri, Phys. Rev. Lett. 108, 136801 (2012).

44 N. Driza, S. Blanco-Canosa, M. Bakr, M. Khalid, L. Mustafa, K. Kawashima, M. C. Hoffman, G. Christiani, H. U. Habermeir, C. Ulrich, M. L. Tacon, and B. Keimer, Nature Mat. 108, 136801 (2012).

45 P. A. Fleury and R. Loudon, Phys. Rev. 166, 514 (1968). 
TABLE III. System $1-$ Normal Modes for the Glazer system $a^{0} a^{0} a^{0}$.

\begin{tabular}{lcccc}
\hline \hline & $A$ & $B$ & $O$ & Selection Rules \\
\cline { 3 - 3 }$a^{0} a^{0} a^{0}$ & $O_{h}(1)$ & $O_{h}(1)$ & $D_{4 h}(3)$ & $T$ \\
\hline$T_{1 u}$ & 1 & 1 & 2 & 1 \\
$T_{2 u}$ & & & 9 & 15 \\
\hline Sum & 3 & 3 & 9 & \\
\hline \hline
\end{tabular}

TABLE IV. System 2-Normal Modes for the Glazer system $a^{0} a^{0} c^{+}$.

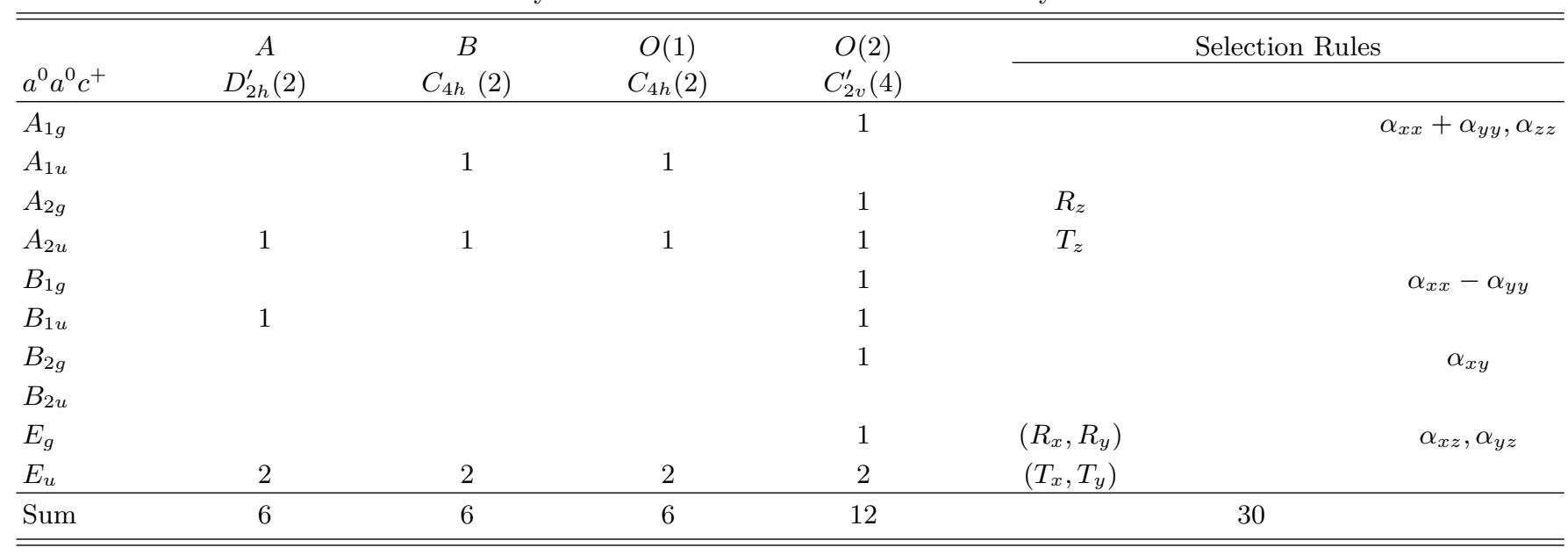

TABLE V. System 3-Normal Modes for the Glazer system $a^{0} b^{+} b^{+}$.

\begin{tabular}{|c|c|c|c|c|c|c|c|c|}
\hline \multirow{2}{*}{$a^{0} b^{+} b^{+}$} & \multirow{2}{*}{$\begin{array}{c}A(1) \\
D_{2 h}(4)\end{array}$} & \multirow{2}{*}{$\begin{array}{c}A(2) \\
D_{4 h}(2)\end{array}$} & \multirow{2}{*}{$\begin{array}{c}A(3) \\
D_{4 h}(2)\end{array}$} & \multirow{2}{*}{$\begin{array}{c}B \\
C_{2 h}^{\prime}(8)\end{array}$} & \multirow{2}{*}{$\begin{array}{c}O(1) \\
C_{s}^{v}(16)\end{array}$} & \multirow{2}{*}{$\begin{array}{c}O(2) \\
C_{2 v}^{\prime}(8) \\
\end{array}$} & \multicolumn{2}{|c|}{ Selection Rules } \\
\hline & & & & & & & & \\
\hline$A_{1 g}$ & & & & & 2 & 1 & & $\alpha_{x x}+\alpha_{y y}, \alpha_{z z}$ \\
\hline$A_{1 u}$ & & & & 1 & 1 & & & \\
\hline$A_{2 g}$ & & & & & 1 & 1 & $R_{z}$ & \\
\hline$A_{2 u}$ & 1 & 1 & 1 & 2 & 2 & 1 & $T_{z}$ & \\
\hline$B_{1 g}$ & & & & & 2 & 1 & & $\alpha_{x x}-\alpha_{y y}$ \\
\hline$B_{1 u}$ & & & & 2 & 1 & 1 & & \\
\hline$B_{2 g}$ & & & & & 1 & 1 & & $\alpha_{x y}$ \\
\hline$B_{2 u}$ & 1 & & & 1 & 2 & & & \\
\hline$E_{g}$ & & & & & 3 & 1 & $\left(R_{x}, R_{y}\right)$ & $\left(\alpha_{x z}, \alpha_{y z}\right)$ \\
\hline$E_{u}$ & 2 & 1 & 1 & 3 & 3 & 2 & $\left(T_{x}, T_{y}\right)$ & \\
\hline Sum & 6 & 3 & 3 & 12 & 24 & 12 & & \\
\hline
\end{tabular}

TABLE VI. System 4-Normal Modes for the Glazer system $a^{+} a^{+} a^{+}$.

\begin{tabular}{|c|c|c|c|c|c|c|}
\hline \multirow[b]{2}{*}{$a^{+} a^{+} a^{+}$} & \multirow{2}{*}{$\begin{array}{l}A(1) \\
T_{h}(2)\end{array}$} & \multirow{2}{*}{$\begin{array}{c}A(2) \\
D_{2 h}(6)\end{array}$} & \multirow{2}{*}{$\begin{array}{c}B \\
S_{6}(8) \\
\end{array}$} & \multirow{2}{*}{$\begin{array}{c}O \\
C_{s}(24)\end{array}$} & \multicolumn{2}{|r|}{ Selection Rules } \\
\hline & & & & & & \\
\hline$A_{g}$ & & & & 2 & & $\alpha_{x x}+\alpha_{y y}+\alpha_{z z}$ \\
\hline$A_{u}$ & & & 1 & 1 & & \\
\hline$E_{g}$ & & & & 2 & & $\left(\alpha_{x x}+\alpha_{y y}-2 \alpha_{z z}, \sqrt{3} \alpha_{x x}-\sqrt{3} \alpha_{y y}\right)$ \\
\hline$E_{u}$ & & & 1 & 1 & & \\
\hline$T_{g}$ & & & & 4 & $R$ & $\alpha_{x x}, \alpha_{x z}, \alpha_{y z}$ \\
\hline$T_{u}$ & 1 & 3 & 3 & 5 & $T$ & \\
\hline Sum & 3 & 9 & 12 & 36 & 60 & \\
\hline
\end{tabular}


TABLE VII. System 5 - Normal Modes for the Glazer system $a^{+} b^{+} c^{+}$.

\begin{tabular}{|c|c|c|c|c|c|c|c|c|c|c|}
\hline \multirow{2}{*}{$a^{+} b^{+} c^{+}$} & \multirow{2}{*}{$\begin{array}{c}A(1) \\
D_{2 h}(2)\end{array}$} & \multirow{2}{*}{$\begin{array}{c}A(2) \\
D_{2 h}(2) \\
\end{array}$} & \multirow{2}{*}{$\begin{array}{c}A(3) \\
D_{2 h}(2)\end{array}$} & \multirow{2}{*}{$\begin{array}{c}A(4) \\
D_{2 h}(2)\end{array}$} & \multirow{2}{*}{$\begin{array}{c}B \\
C_{i}(8)\end{array}$} & \multirow{2}{*}{$\begin{array}{c}O(1) \\
C_{s}^{y z}(8)\end{array}$} & \multirow{2}{*}{$\begin{array}{c}O(2) \\
C_{s}^{x y}(8) \\
\end{array}$} & \multirow{2}{*}{$\begin{array}{c}O(3) \\
C_{s}^{x z}(8)\end{array}$} & \multicolumn{2}{|r|}{ Selection Rules } \\
\hline & & & & & & & & & & \\
\hline$A_{g}$ & & & & & & 2 & 2 & 2 & & $\alpha_{x x}, \alpha_{y y}, \alpha_{z z}$ \\
\hline$A_{u}$ & & & & & 3 & 1 & 1 & 1 & & \\
\hline$B_{1 g}$ & & & & & & 1 & 2 & 1 & $R_{z}$ & $\alpha_{x y}$ \\
\hline$B_{1 u}$ & 1 & 1 & 1 & 1 & 3 & 2 & 1 & 2 & $T_{z}$ & \\
\hline$B_{2 g}$ & & & & & & 1 & 1 & 2 & $R_{y}$ & $\alpha_{x z}$ \\
\hline$B_{2 u}$ & 1 & 1 & 1 & 1 & 3 & 2 & 2 & 1 & $T_{y}$ & \\
\hline$B_{3 g}$ & & & & & & 2 & 1 & 1 & $R_{x}$ & $\alpha_{y z}$ \\
\hline$B_{3 u}$ & 1 & 1 & 1 & 1 & 3 & 1 & 2 & 2 & $T_{x}$ & \\
\hline Sum & 3 & 3 & 3 & 3 & 12 & 12 & 12 & 12 & & 60 \\
\hline
\end{tabular}

TABLE VIII. System 6 - Normal Modes for the Glazer system $a^{0} a^{0} c^{-}$.

\begin{tabular}{|c|c|c|c|c|c|c|}
\hline \multirow{2}{*}{$a^{0} a^{0} c^{-}$} & \multirow{2}{*}{$\begin{array}{c}A \\
D_{2 d}^{\prime}(4) \\
\end{array}$} & \multirow{2}{*}{$\begin{array}{c}B \\
C_{4 h}(4) \\
\end{array}$} & \multirow{2}{*}{$\begin{array}{c}O(1) \\
C_{2 v}^{\prime}(8) \\
\end{array}$} & \multirow{2}{*}{$\begin{array}{c}O(2) \\
D_{4}(4) \\
\end{array}$} & \multicolumn{2}{|c|}{ Selection Rules } \\
\hline & & & & & & \\
\hline$\overline{A_{1 g}}$ & & & 1 & & & $\alpha_{x x}+\alpha_{x x}, \alpha_{z z}$ \\
\hline$A_{1 u}$ & & 1 & & & & \\
\hline$A_{2 g}$ & & & 1 & 1 & $R_{z}$ & \\
\hline$A_{2 u}$ & 1 & 1 & 1 & 1 & $T_{z}$ & \\
\hline$B_{1 g}$ & & & 1 & & & $\alpha_{x x}-\alpha_{y y}$ \\
\hline$B_{1 u}$ & & & 1 & & & \\
\hline$B_{2 g}$ & 1 & & 1 & & & $\alpha_{x y}$ \\
\hline \multicolumn{7}{|l|}{$B_{2 u}$} \\
\hline$E_{g}$ & 1 & & 1 & 1 & $\left(R_{x}, R_{y}\right)$ & $\left(\alpha_{x z}, \alpha_{y z}\right)$ \\
\hline$E_{u}$ & 1 & 2 & 2 & 1 & $\left(T_{x}, T_{y}\right)$ & \\
\hline Sum & 6 & 6 & 12 & 6 & & \\
\hline
\end{tabular}

TABLE IX. System 7-Normal Modes for the Glazer system $a^{0} b^{-} b^{-}$.

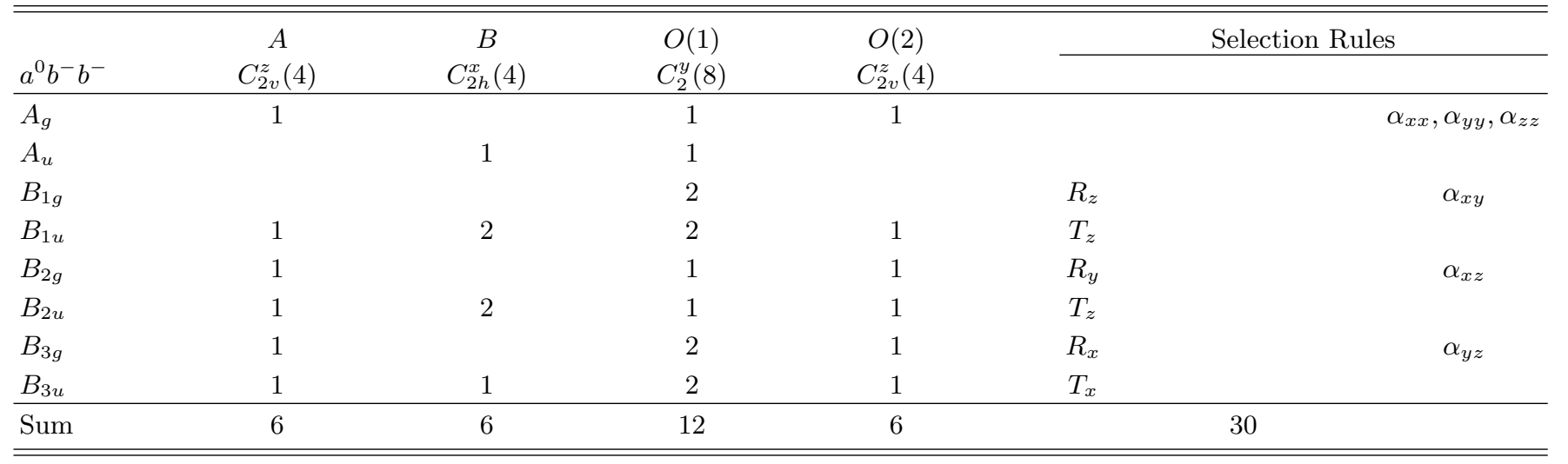


TABLE X. System 8-Normal Modes for the Glazer system $a^{-} a^{-} a^{-}$.

\begin{tabular}{lccccc}
\hline \hline & $A$ & $B$ & $O$ & & Selection Rules \\
\cline { 3 - 4 }$a^{-} a^{-} a^{-}$ & $D_{3}(2)$ & $S_{6}(2)$ & $C_{2}(6)$ & \\
\hline$A_{1 g}$ & & & 1 & $\alpha_{x x}, \alpha_{y y}, \alpha_{z z}$ \\
$A_{1 u}$ & 1 & 1 & 1 & $R_{z}$ & $\left(\alpha_{x x}-\alpha_{y y}, \alpha_{x y}\right),\left(\alpha_{x z}, \alpha_{y z}\right)$ \\
$A_{2 g}$ & 1 & & 2 & $T_{z}$ & $\left(R_{x}, R_{y}\right)$ \\
$A_{2 u}$ & 1 & 1 & 2 & $T_{x}, T_{y}$ & 30 \\
$E_{g}$ & 1 & & 3 & & \\
$E_{u}$ & 6 & 2 & 1 & 18 & \\
\hline Sum & 6 & &
\end{tabular}

TABLE XI. System $9-$ Normal Modes for the Glazer system $a^{0} b^{-} c^{-}$.

\begin{tabular}{lccccccc}
\hline \hline & $A_{1}$ & $B$ & $O(1)$ & $O(2)$ & $O(3)$ & & Selection Rules \\
\cline { 5 - 7 }$a^{0} b^{-} c^{-}$ & $C_{s}(4)$ & $C_{i}(4)$ & $C_{2}(4)$ & $C_{2}(4)$ & $C_{s}(4)$ & $R_{z x}, \alpha_{y y}, \alpha_{z z}, \alpha_{x y}$ \\
\hline$A_{g}$ & 2 & & 1 & 1 & 2 & $T_{z}$ & $\alpha_{x x}, \alpha_{y y}$ \\
$A_{u}$ & 1 & 3 & 1 & 1 & 1 & $R_{x}, R_{y}$ & 30 \\
$B_{g}$ & 1 & & 2 & 2 & 1 & $T_{x}, T_{y}$ \\
$B_{u}$ & 2 & 3 & 2 & 2 & 6 & 6 \\
\hline Sum & 6 & 6 & 6 & 6 & 6 & \\
\hline \hline
\end{tabular}

TABLE XII. System 10-Normal Modes for the Glazer system $a^{-} b^{-} b^{-}$.

\begin{tabular}{lcccccc}
\hline \hline & $A_{1}$ & $B$ & $O(1)$ & $O(2)$ & & Selection Rules \\
\cline { 5 - 6 }$a^{-} b^{-} b^{-}$ & $C_{2}(4)$ & $C_{i}(4)$ & $C_{1}(8)$ & $C_{2}(4)$ & & $\alpha_{x x}, \alpha_{y y}, \alpha_{z z}, \alpha_{x y}$ \\
\hline$A_{g}$ & 1 & & 3 & 1 & $R_{z}$ & $\alpha_{x x}, \alpha_{y y}$ \\
$A_{u}$ & 1 & 3 & 3 & 1 & $T_{z}$ & $R_{x}, R_{y}$ \\
$B_{g}$ & 2 & & 3 & 2 & $T_{x}, T_{y}$ & 30 \\
$B_{u}$ & 2 & 3 & 3 & 2 & & \\
\hline Sum & 6 & 6 & 12 & 6 & & \\
\hline \hline
\end{tabular}

TABLE XIII. System $11-$ Normal Modes for the Glazer system $a^{-} b^{-} c^{-}$.

\begin{tabular}{lcccccccc}
\hline \hline & $A$ & $B(1)$ & $B(2)$ & $O(1)$ & $O(2)$ & $O(2)$ & Selection Rules \\
\cline { 3 - 7 }$a^{-} b^{-} c^{-}$ & $C_{1}(2)$ & $C_{i}(1)$ & $C_{i}(1)$ & $C_{1}(2)$ & $C_{1}(2)$ & $C_{1}(2)$ & $R$ \\
\hline$A_{g}$ & 3 & & & 3 & 3 & 3 & $T$ \\
$A_{u}$ & 3 & 3 & 3 & 3 & 3 & 3 & $T$ \\
\hline Sum & 6 & 3 & 3 & 6 & 6 & 6 & 30 \\
\hline \hline
\end{tabular}


TABLE XIV. System 12-Normal Modes for the Glazer system $a^{0} b^{+} c^{-}$.

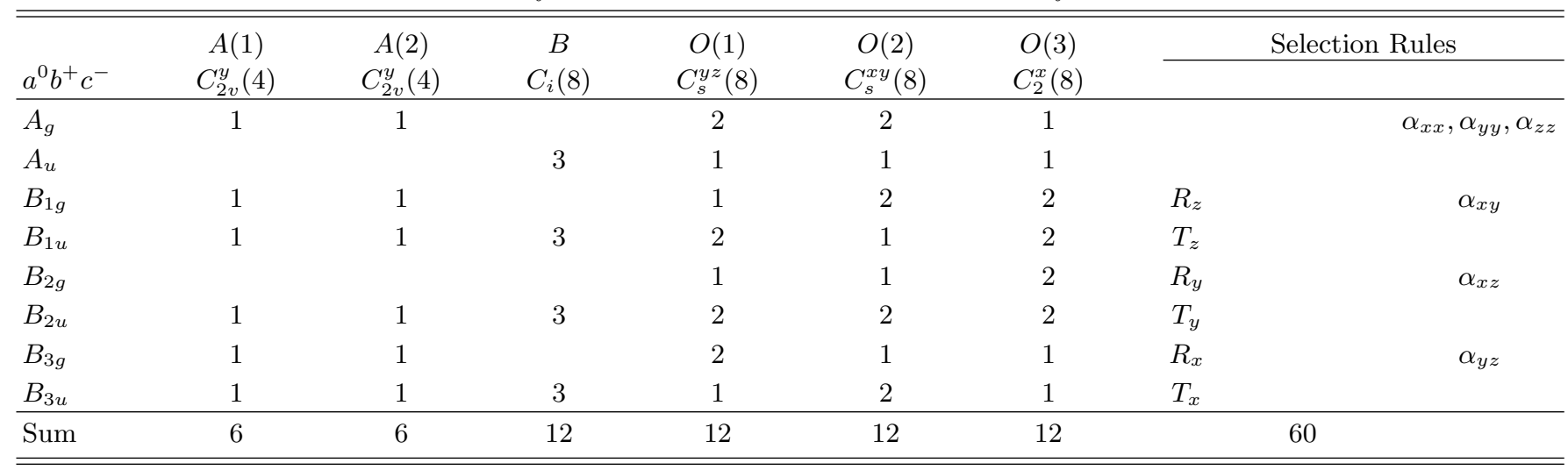

TABLE XV. System 13-Normal Modes for the Glazer system $a^{+} b^{-} b^{-}$.

\begin{tabular}{|c|c|c|c|c|c|c|}
\hline \multirow{2}{*}{$a^{+} b^{-} b^{-}$} & \multirow{2}{*}{$\begin{array}{c}A \\
C_{s}^{y z}(4)\end{array}$} & \multirow{2}{*}{$\begin{array}{c}B \\
C_{i}(4) \\
\end{array}$} & \multirow{2}{*}{$\begin{array}{c}O(1) \\
C_{s}^{y z}(4)\end{array}$} & \multirow{2}{*}{$\begin{array}{l}O(2) \\
C_{1}(8) \\
\end{array}$} & \multicolumn{2}{|c|}{ Selection Rules } \\
\hline & & & & & & \\
\hline$A_{g}$ & 2 & & 2 & 3 & & $\alpha_{x x}, \alpha_{y y}, \alpha_{z z}$ \\
\hline$A_{u}$ & 1 & 3 & 1 & 3 & & \\
\hline$B_{1 g}$ & 1 & & 1 & 3 & $R_{z}$ & $\alpha_{x y}$ \\
\hline$B_{1 u}$ & 2 & 3 & 2 & 3 & $T_{z}$ & \\
\hline$B_{2 g}$ & 2 & & 2 & 3 & $R_{y}$ & $\alpha_{x z}$ \\
\hline$B_{2 u}$ & 1 & 3 & 1 & 3 & $T_{y}$ & \\
\hline$B_{3 g}$ & 1 & & 1 & 3 & $R_{x}$ & $\alpha_{y z}$ \\
\hline$B_{3 u}$ & 2 & 3 & 2 & 3 & $T_{x}$ & \\
\hline Sum & 12 & 12 & 12 & 24 & & \\
\hline
\end{tabular}

TABLE XVI. System 14-Normal Modes for the Glazer system $a^{+} b^{-} c^{-}$.

\begin{tabular}{lcccccccccc}
\hline \hline & $A(1)$ & $A(2)$ & $B(1)$ & $B(2)$ & $O(1)$ & $O(2)$ & $O(3)$ & $O(4)$ & Selection Rules \\
\cline { 5 - 9 }$a^{+} b^{-} c^{-}$ & $C_{s}(2)$ & $C_{s}(2)$ & $C_{i}(2)$ & $C_{i}(2)$ & $C_{1}(4)$ & $C_{s}(2)$ & $C_{s}(2)$ & $C_{1}(4)$ & $R_{z x}, \alpha_{y y}, \alpha_{z z}, \alpha_{x y}$ \\
\hline$A_{g}$ & 2 & 2 & & & 3 & 2 & 2 & 3 & $R_{z}$ \\
$A_{u}$ & 1 & 1 & 3 & 3 & 3 & 1 & 1 & 3 & $T_{z}$ & $\alpha_{x x}, \alpha_{y y}$ \\
$B_{g}$ & 1 & 1 & & & 3 & 1 & 1 & 3 & $R_{x}, R_{y}$ & $T_{x}, T_{y}$ \\
$B_{u}$ & 2 & 2 & 3 & 3 & 3 & 2 & 2 & 3 & 60 \\
\hline Sum & 6 & 6 & 6 & 6 & 12 & 6 & 6 & 12 & \\
\hline \hline
\end{tabular}


TABLE XVII. System 15-Normal Modes for the Glazer system $a^{+} a^{+} c^{-}$.

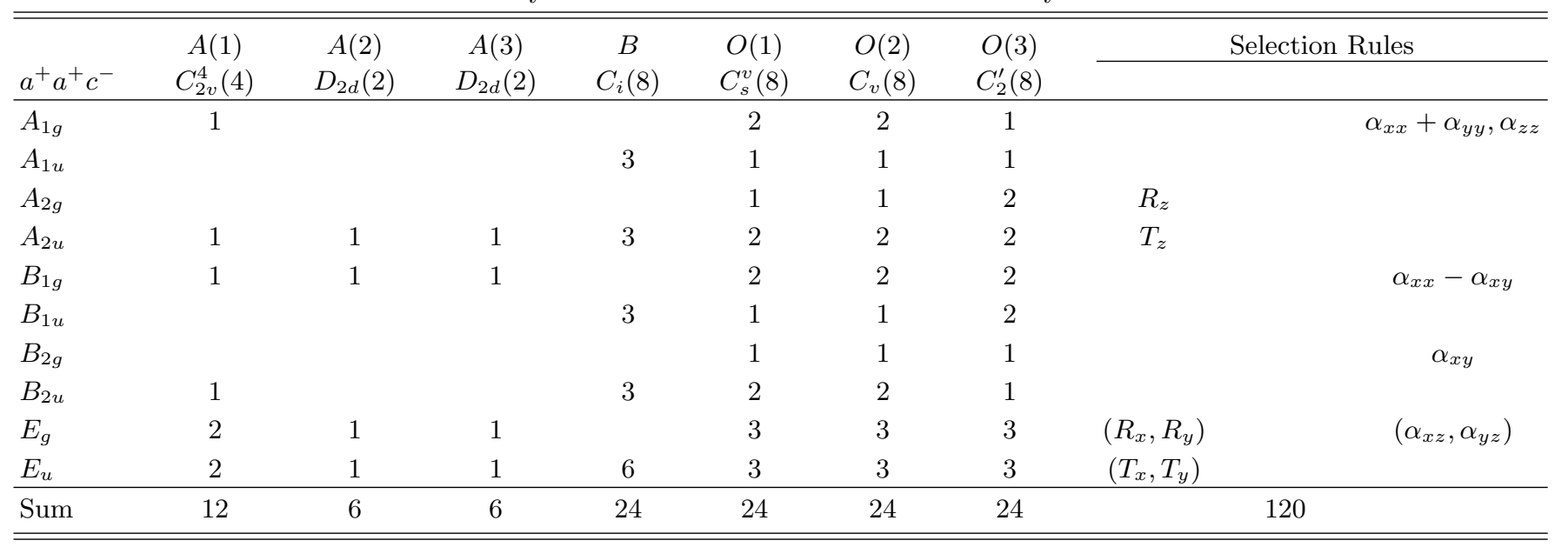

TABLE XVIII. System 16-Normal Modes for the Non Glazer system $\mathrm{BiFeO}_{3} a^{-} a^{-} a^{-}$.

\begin{tabular}{lccccc}
\hline \hline & $B$ & $F e$ & $O$ & & Selection Rules \\
\cline { 4 - 6 }$a^{-} a^{-} a^{-}$ & $C_{3}(2)$ & $C_{3}(2)$ & $C_{1}(6)$ & $T_{z}$ & $\alpha_{x x}^{z}+\alpha_{y y}^{z}, \alpha_{z z}^{z}$ \\
\hline$A_{1}$ & 1 & 1 & 3 & $R_{z}$ & $\left(\alpha_{x x}^{y}-\alpha_{y y}^{y}, \alpha_{x y}^{x}\right),\left(\alpha_{x z}^{x}, \alpha_{y z}^{y}\right)$ \\
$A_{2}$ & 1 & 1 & 3 & $\left(T_{x}, T_{y}\right) ;\left(R_{x} R_{y}\right)$ & 30 \\
$E$ & 2 & 2 & 6 & & \\
\hline Sum & 6 & 6 & 18 & \\
\hline \hline
\end{tabular}

